\title{
Treatment of hospital-acquired pneumonia with multi-drug resistant organism by Buzhong Yiqi decoction based on Fuzheng Quxie classical prescription: study protocol for a randomized controlled trial
}

Dong Deng ${ }^{1}$, Zhenyi Chen ${ }^{1,2}$, Liyang Jia', Jianhong Bu ${ }^{1,3}$, Miaoqing Ye ${ }^{1,4}$, Lihua Sun ${ }^{1}$, Yun Gen ${ }^{1}$, Wen Zhang ${ }^{1}$, Gang Chen ${ }^{1,5}$ and Bangjiang Fang ${ }^{1 *}$ (D)

\begin{abstract}
Background: Drug resistance in China is becoming a more and more serious issue. Infection by drug-resistant bacteria has become a major disease that seriously threatens the health of Chinese people and affects national medical finance. Therefore, it is of great scientific and clinical significance to actively carry out research on the prevention and treatment of infections by multi-drug resistant organisms (MDRO). Previous studies by the authors suggested that patients with hospital-acquired pneumonia caused by MDRO mostly showed the pathological state of "insufficient healthy Qi and internal accumulation of pathogenic Qi" and "acute deficiency syndrome" mainly characterized by Qi deficiency. Buzhong Yiqi decoction is a famous classic prescription in traditional Chinese medicine (TCM) for treating internal damage fever. This study intends to provide an evidence-based rationale for Buzhong Yiqi decoction in treating MDRO hospital-acquired pneumonia by conducting a multi-center randomized controlled clinical study.

Methods/design: This study is designed to be a multi-center randomized controlled study in which patients are assigned randomly into control (standard therapy) and trial (standard therapy plus Buzhong Yiqi decoction) groups. The patients will be selected from the emergency department and the ICU inpatient department of five study sites and will all be diagnosed with MDRO hospital-acquired pneumonia and meet the inclusion criteria. Forty patients are to be enrolled in each study site, resulting in a total of 200 patients in the study. The treatment course is 28 days.

Discussion: In this study: (1) the theory of "acute Qi deficiency" in MDRO hospital-acquired pneumonia is put forward for the first time, and the basic theories of TCM are further improved; (2) a multi-center randomized controlled clinical study will be performed for the first time with Buzhong Yiqi decoction, the classic prescription for reinforcing healthy Qi and eliminating pathogenic Qi, providing a reliable evidence-based rationale for the treatment of MDRO pulmonary infection with TCM; (3) the clinical application and modern disease spectrum of Buzhong Yiqi decoction is expanded, and the scientific notion of "treating different diseases with the same method" is enriched further.
\end{abstract}

Trial registration: China Clinical Trial Registry, ChiCTR1900022429. Registered on April 11, 2019. http://www.chictr.org. cn/listbycreater.aspx.

Keywords: Hospital acquired pneumonia caused by multi-drug resistant organisms, Buzhong Yiqi decoction, Efficacy, Multicenter, Randomized controlled clinical study

\footnotetext{
* Correspondence: fangbji@163.com

${ }^{1}$ LongHua Hospital Shanghai University of Traditional Chinese Medicine,

NO.725 Wanping South Road, Xuhui District, Shanghai 200032, China

Full list of author information is available at the end of the article
}

(c) The Author(s). 2019 Open Access This article is distributed under the terms of the Creative Commons Attribution 4.0 International License (http://creativecommons.org/licenses/by/4.0/), which permits unrestricted use, distribution, and reproduction in any medium, provided you give appropriate credit to the original author(s) and the source, provide a link to the Creative Commons license, and indicate if changes were made. The Creative Commons Public Domain Dedication waiver (http://creativecommons.org/publicdomain/zero/1.0/) applies to the data made available in this article, unless otherwise stated. 


\section{Background}

Clinical studies on hospital-acquired pneumonia (HAP) and/or ventilator-associated pneumonia (VAP) have detected multi-drug-resistant organisms (MDROs) in most respiratory secretions from patients in intensive care units (ICUs), with the isolation rate increasing year on year [1-4]. In recent years, the national bacterial resistance surveillance report has revealed that MDROs have, to varying degrees, serious epidemic characteristics in different provinces of China. The increasing social burden of MDRO infections arises from adverse clinical outcomes and aggravated economic and medical burdens [5-7].

HAP caused by MDRO infection is a common and major clinical disease, especially in emergency departments and ICUs [8]. According to its clinical manifestations, the disease can generally be classified as "pulmonary fever" in traditional Chinese medicine. Our previous clinical studies found that HAP caused by MDROs, due to the underlying and excessive pathogenic factors prevailing over the normal resistance of the body, first showed the signs of $Q i$ deficiency, especially weakness of the spleen and stomach, giving rise to a pathological state of mainly "acute Qi deficiency". Combining with theories from traditional Chinese medicine (TCM), we innovatively put forward a theory of "acute deficiency syndrome" in critical and severe cases of HAP caused by MDRO infection. Based on studies by other scholars on TCM syndrome types of MDRO HAP [9-12], we believe that the core pathogenesis of MDRO HAP is "insufficient healthy $Q i$ and internal accumulation of pathogenic $Q i$ ", in which "acute Qi deficiency syndrome" is critical.

Buzhong Yiqi decoction was proposed in On Spleen and Stomach by Li Dongyuan, one of the four greatest medical scientists in the Jin and Yuan Dynasties. It is a famous classical prescription in TCM and also a typical representative prescription in treating internal damage fever. According to TCM, "Pathogenic Qi cannot invade the body with sufficient healthy $Q i$ inside" and disease occurs when healthy $Q i$ is weak. Supplementing pectoral $Q i$ is the method to cure the root cause, and abundance of pectoral $Q i$ depends on the lung and spleen. Li's Buzhong Yiqi decoction was formulated considering the methods of "treating impairment with supplementing" and "treating overstrain with warming" in The Yellow Emperor's Canon of Internal Medicine, pioneering the school of "relieving great fever with drugs sweet in taste and warm in property". This prescription consists of Radix Astragali seu Hedysari, Radix Ginseng, Radix Glycyrrhiza, Rhizoma Atractylodis Macrocephalae, Radix Angelicae Sinensis, Pericarpium Citri Reticulatae, Rhizoma Cimicifugae, and Radix Bupleuri. In the prescription, Radix Astragali seu Hedysari is the sovereign medicinal that invigorates $Q i$, Radix Ginseng is the minister medicinal that powerfully tonifies the primordial Qi, nourishes the lung, and invigorates the spleen, Rhizoma Atractylodis Macrocephalae invigorates the spleen, Radix Angelicae Sinensis regulates the blood, Pericarpium Citri Reticulatae regulates Qi, and prepared Radix Glycyrrhiza helps Radix Ginseng and Radix Astragali seu Hedysari rectify Qi; Rhizoma Cimicifugae and Radix Bupleuri have the function of eliminating pathogens and raising the clear yang. The whole formula supplements and clears in that it not only tonifies the middle and replenishes $Q i$, but also unblocks [13-16].

Clinical pharmacological studies with Buzhong Yiqi decoction have demonstrated that it enhances phagocytosis of the reticuloendothelial system, promotes the non-specific immune function of the body, and has a remarkable inhibitory effect on multi-drug-resistant Staphylococcus aureus $[17,18]$. This recipe can effectively alleviate inflammatory reactions and boost immunity of the body. Results from previous studies by the authors on Buzhong Yiqi decoction for the treatment of HAP, including that caused by MDROs, showed that TCM could significantly relieve the inflammatory reactions of patients, improve clearance of the organisms, shorten the time of mechanical ventilation, and increase the success rate of weaning from the ventilator and had good clinical efficacy. Similar clinical efficacy was observed in other related studies on Buzhong Yiqi decoction for the treatment of HAP [19-22]. Therefore, on the basis of previous studies, this study intends to provide an evidence-based rationale and guidance for the clinical treatment of MDRO HAP, further elucidate the scientific notion of "treating different diseases with the same method", and expand the modern disease spectrum of Buzhong Yiqi decoction by performing a multi-center randomized controlled clinical study with Buzhong Yiqi decoction (a classical formula for reinforcing healthy $Q i$ and eliminating pathogenic $Q i$ ) in the treatment of MDRO HAP.

\section{Methods/design Objective}

We propose a multi-center randomized controlled clinical study to demonstrate the efficacy and safety of Buzhong Yiqi decoction in the treatment of MDRO HAP and to explore the possible mechanism, provide a reliable evidencebased rationale for the treatment of MDRO pulmonary infection with TCM, further elucidate the scientific notion of "treating different diseases with the same method", and expand the modern disease spectrum of Buzhong Yiqi decoction. We do this to open up new ideas and methods for MDRO prevention and treatment strategies and formulate TCM diagnosis and treatment regimens for pulmonary infections caused by MDROs in clinical practice.

\section{Design}

This study is a multi-center randomized controlled study in which patients will be assigned randomly into control 
and trial groups. The patients will be selected from the emergency department and the ICU inpatient department of five study sites after being diagnosed with MDRO HAP and meeting the inclusion criteria. The flow chart of the study is shown in Fig. 1. The date of study initiation was July 1, 2018 and the date of study completion is June 30, 2021. The Standard Protocol Items: Recommendations for Interventional Trials (SPIRIT) Checklist can be found in Additional file 1.

\section{Participants}

From July 1, 2018 to May 31, 2021, patients will be selected from those treated in the emergency department and the ICU inpatient department of the five study sites, namely Longhua Hospital Shanghai University of Traditional Chinese Medicine, Shanghai Integrated Traditional Chinese and Western Medicine Hospital, Shanghai Seventh People's Hospital, Shanghai Baoshan Integrated Traditional Chinese and Western Medicine Hospital, and Shanghai Pudong New Area Public Interest Hospital. All patients are diagnosed with MDRO HAP and meet the inclusion criteria. Forty patients are to be enrolled in each study site, resulting in a total of 200 patients in the study.

\section{Inclusion criteria}

The eligibility criteria are as follows: (1) patients meet the diagnostic criteria of Western medicine; (2) etiological diagnosis meets the MDRO criteria; (3) aged 40-85 years; (4) patients have signed the informed consent form; (5) Patients also meeting the following criteria as Exclusion criteria, withdrawal/trial discontinuation/drop-out criteria are included into observation.

\section{Exclusion criteria}

The exclusion criteria are as follows: (1) severe primary or malignant disease of the heart, brain, liver, kidney, or other organs or systemic disease at an acute or progressive stage; (2) surgery within 2 months accompanied by surgical site infection; (3) patients who have been hospitalized less than $72 \mathrm{~h}$ or who die within $72 \mathrm{~h}$ of hospitalization; (4) psychiatric patients and pregnant women; (5) active tuberculosis; (6) a history of allergy to on-treatment drugs; (7) recent drug and alcohol abuse; (8) patients who receive no other antibiotics or fail to respond to other antibiotics within 3 months prior to the clinical trial.

\section{Withdrawal/trial discontinuation/drop-out criteria}

The rejection and withdrawal criteria are: (1) not meeting the inclusion criteria of the study; (2) incomplete clinical data obtained after inclusion and it is impossible to perform further clinical statistical analyses; (3) subjects experience severe adverse events/reactions related to the treatment regimen and the investigator considers it necessary to withdraw them from the trial; (4) during the trial, the patient's condition continues to deteriorate and dangerous events are likely to occur such that the investigator considers it necessary to withdraw them from the clinical trial; (5) non-specified combination of drugs, especially those that have a great impact on the observational drug and affect efficacy and safety assessment; (6) patients who withdraw during the trial at their own discretion-all patients who complete the informed consent form and are eligible to enter the trial at screening, no matter when and why they withdraw, are classified as drop-out cases as long as they do not complete the observational period specified in the protocol; (7) poor treatment compliance that affects determination of efficacy and safety.

\section{Ethics}

This trial is conducted in accordance with the Helsinki Declaration and Chinese Good Clinical Practice and relevant regulations and has been approved by the Medical

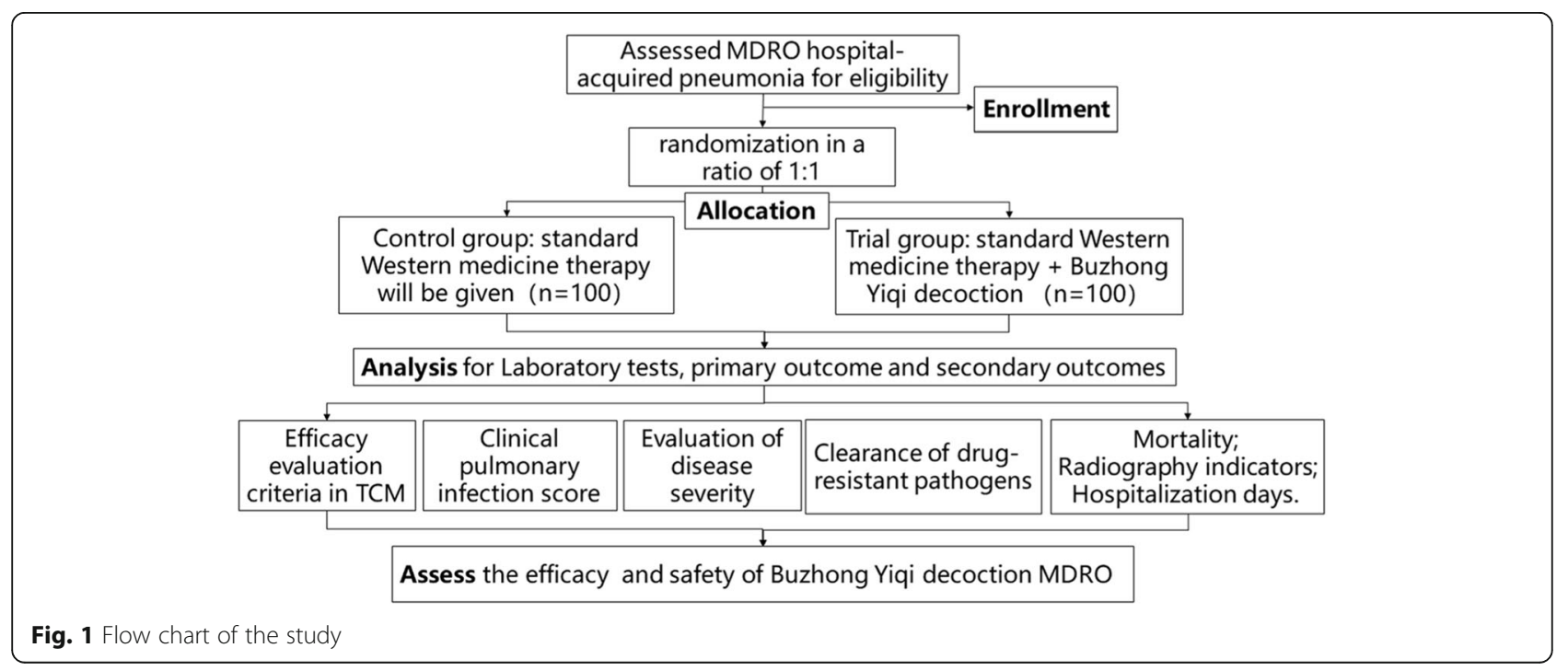


Ethics Committee of Longhua Hospital Shanghai University of Traditional Chinese Medicine. To protect the privacy of subjects, data processing is performed anonymously and written informed consent is obtained from each patient before initiation of the clinical trial.

\section{Randomization and allocation}

In each study site, patients with MDRO HAP that fulfill the inclusion criteria and do not meet any of the exclusion criteria are assigned to the trial group or control group according to the corresponding random numbers extracted in the order at visit. Each study site will enroll 40 patients for a total of 200 over the five study sites. A random number table generated using Statistical Analysis Software (SAS), version 9.2, using the randomization method, will be used to assign the participants in a ratio of 1:1, with 100 patients each in the trial group and control group. A researcher will generate the allocation sequence and enroll participants and the clinical researchers will assign participants to interventions. The clinical researchers at each center will provide packaged drugs to the participants according to the randomization number on the packaging; the code labeling will conform to the principles of GCP. The statistician will uncover the blinding as necessary.

\section{Interventions}

The treatment course is 28 days.

The control group will be given standard Western medicine therapy, including: routine monitoring of vital signs; blood, arterial blood gas, and biochemical analysis; coagulation monitoring; step-down antimicrobial therapy; handle the respiratory secretions; mechanical ventilation; liquid nutrient support; maintenance of blood sugar, acid base, and electrolyte balance; correct coagulation function; etc.

The trial group will receive standard Western medicine therapy plus Buzhong Yiqi decoction (Radix Astragali seu Hedysari 45 g, Radix Codonopsis $15 \mathrm{~g}$, fried Rhizoma Atractylodis Macrocephalae $15 \mathrm{~g}$, prepared Radix Glycyrrhiza 15 g, Radix Angelicae Sinensis $10 \mathrm{~g}$, Pericarpium Citri Reticulatae $9 \mathrm{~g}$, Rhizoma Cimicifugae $12 \mathrm{~g}$, and Radix Bupleuri $20 \mathrm{~g}$; NONG's granular preparation, dissolved in $200 \mathrm{~mL}$ warm boiled water and taken orally or by nasal feed once daily).

\section{Outcome measures}

\section{Laboratory tests}

In both groups laboratory tests will include routine blood, urine, and fecal tests, blood gas analysis, liver and kidney function, coagulation function, and lactic acid, Creactive protein, and procalcitonin analysis.

\section{Primary outcome}

\section{Efficacy evaluation criteria in TCM}

Evaluation criteria by clinical manifestations are in reference to the Criteria of Diagnosis and Therapeutic Effect of Diseases and Syndromes in Traditional Chinese Medicine (Chinese Medicine Industry Standards of the People's Republic of China; ZY/T001.1-94):

1. Clinical control: After treatment, symptoms and signs almost disappear and patients return to normal activities and work. The efficacy rate for syndromes is $\geq 90 \%$.

2. Excellent efficacy: After treatment, symptoms and signs and the results of examinations improve significantly. The efficacy rate for syndromes is $\geq 60 \%$ but $<90 \%$.

3. Efficacy: After treatment, symptoms and signs and the results of examinations improve. The efficacy rate for syndromes is $\geq 30 \%$ but $<60 \%$.

4. Lack of efficacy: After treatment, symptoms and signs and the results of examinations do not improve compared to before treatment. The efficacy rate for syndromes is $<30 \%$.

\section{Secondary outcomes}

1. Clinical pulmonary infection score (CPIS): CPIS is a comprehensive clinical and imaging standard to assess the severity of pulmonary infection. It includes the following indicators: body temperature, white blood cell count, tracheal secretions, oxygenation, X-ray pulmonary infiltration, and progress in etiology. The clinical pulmonary infection score is compared before and after treatment. The CPIS details can be found in Additional file 3.

2. Evaluation of disease severity: the scores of patients in the two groups are compared using the APECHA II scoring system before and after treatment. Improvement rate in APECHA II score $=($ Post-treatment score - Pre-treatment score)/Pre-treatment score. The APECHAII details can be found in Additional file 2.

3. Clearance of drug-resistant pathogens: observed on days $7,14,21$, and 28.

4. Comparison of mechanical ventilation time and success rate of weaning off the ventilator are calculated if appropriate.

5. Comparison of mortality: mortality is compared on day 28 between the groups.

6. Radiography indicators: pulmonary CT imaging results are compared between the groups before and after treatment.

7. Number of hospitalization days: survival days of patients who die and hospitalization days of those who survive and are discharged.

Data collection and management

Data will be managed using the electronic data capture (EDC) system. The study protocol and case report form 
(CRF)-related materials are provided by the research team to a professional institution for clinical data management and study data statistics, which will establish an electronic case report form (eCRF) for the team, set up a personalized database for data recording, and is responsible for management of the study site's EDC system. The Standard Protocol Items: Recommendations for Interventional Trials (SPIRIT) flowchart of the trial is shown in Fig. 2.

\section{Data monitoring}

During the study, an independent data monitoring committee (DMC) will be set up to carry out periodic interim evaluation and optimize the study when appropriate based on the results of the interim evaluation. The DMC is authorized to discontinue the clinical study in case of unexpected adverse reactions. During implementation of the project, the original trial data will be subject to audit and random inspection periodically or irregularly and study compliance will be checked, so that data integrity and accuracy will be fully guaranteed and authenticity and reliability of the study results are ensured.

\section{Adverse events}

Any untoward medical event that occurs in subjects during observation of a clinical study, whether or not it is causally related to the investigational drug, is considered an adverse event (AE). The $\mathrm{AE}$ report form is to be completed during the trial. The time of occurrence, severity, duration, actions taken, and outcomes of AEs are recorded. AEs occurring during follow-up should be reported in a timely manner to the sponsor; in case of serious AEs, the events should be reported to the adverse drug reactions (ADR) monitoring center of the local authority within $24 \mathrm{~h}$, and to the sponsor at the same time.

\section{Sample size}

Considering that no previous studies have been conducted to calculate the sample size, and assuming that the withdrawal rate is less than $15 \%$ [23], we expect that 100 patients will be enrolled in each arm, and that the final sample size of each arm will be at least 85 . The data we collect will be helpful to calculate the appropriate sample size in the future and provide reference for further large-scale research.

\section{Statistical analysis \\ Data analysis}

The Longhua Hospital Shanghai University of Traditional Chinese Medicine will be in charge of data management and statistical analysis in this study. Statistical analysts are not involved in clinical observation; they are responsible for statistical analysis of study data and timely delivery of statistical reports to the study director. Statistical analysis will be performed with SPSS 20.0 software. Measurement data are expressed as mean \pm standard deviation. Normality test and

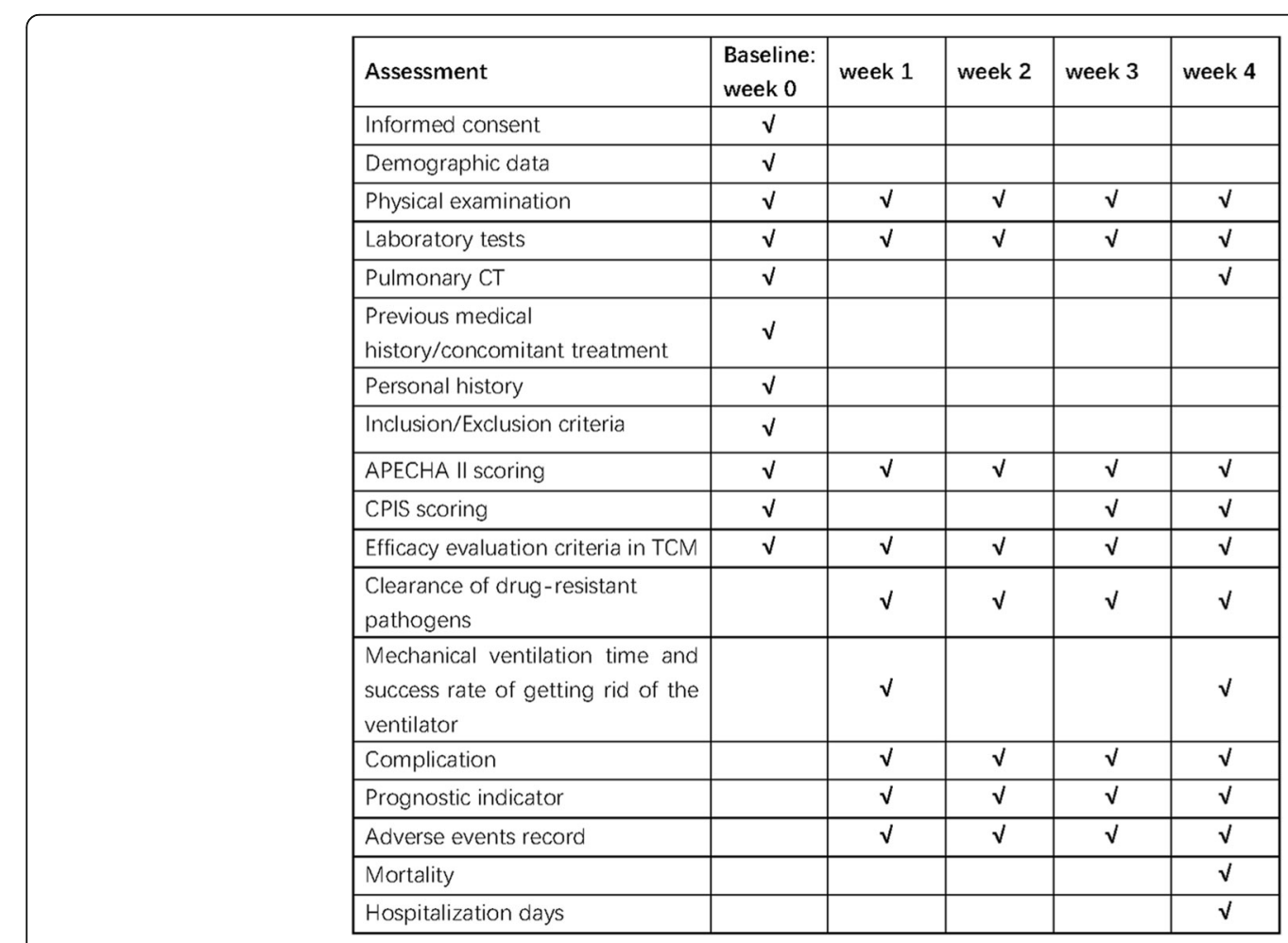

Fig. 2 Standard Protocol Items: Recommendations for Interventional Trials (SPIRIT) flowchart 
homogeneity test of variance will be performed first. In case of normal distribution and homogeneity of variance, a $t$-test is performed, otherwise a non-parametric test is used. Count data are expressed as frequency constituent ratio (percentage) and $X^{2}$ test is performed. $P<0.05$ indicates statistical significance.

\section{Consent}

Patients who meet the study requirements will be offered a consent form covering the study name, registered information, research background, how the study is to be conducted, what participants should do in the study, inclusion/exclusion criteria, treatment plans and obligations, possible drug-related side effects, expenses during participation, etc. We will make every effort to protect the privacy of patients' personal medical data to the extent permitted by law. Participation in this study is completely voluntary, and other treatment options will be offered to patients who do not participate or drop out. When patients sign the informed consent form, their personal and medical information will be used in this study.

\section{Confidentiality}

Participants' medical records will be kept at the hospital, and the investigator, the research authority, and the ethics committee will be allowed access to these medical records. Any public report on the results of this study will not disclose participants' personal identities. We will make every effort to protect the privacy of participants' personal medical data to the extent permitted by law. Personal and medical information will be kept confidential in a safe and reliable place. At any time, participants may request access to their personal information (such as address, contact information, etc.) and may modify this information if necessary.

\section{Discussion}

Infections caused by drug-resistant bacteria have become a major problem that seriously threatens the health of Chinese people and impacts national medical finance. It is of great scientific and clinical significance to actively carry out research on the prevention and treatment of MDRO infections. With the extensive use of broad-spectrum antibiotics in clinical practice, the problem of bacterial resistance is becoming more and more serious [24-26]. The Enterobacteriaceae in an Indian outbreak in 2010 were resistant to all the available antibiotics, which attracted global attention [27]. On January 29, 2018, the World Health Organization (WHO) published monitoring data on bacterial resistance to antibiotics for the first time, which revealed that bacterial resistance was widespread, with serious bacterial infections and high drug resistance in both high- and low-income countries, and had become a major public health problem worldwide [28].
By analyzing the clearance of drug-resistant pathogens, APECHA II score, CPIS score, TCM diagnostic and therapeutic criteria, mortality and related physical and chemical indicators, this study may confirm the clinical safety and efficacy of Buzhong Yiqi decoction in the treatment of MDRO HAP. In addition, by analyzing CRP, platelets, blood lactic acid, procalcitonin, and coagulation function, we will reveal the possible anti-inflammatory mechanism of Buzhong Yiqi decoction in treating MDRO HAP. Finally, if the hypothesis of this study is proved to be true, it can not only provide a clinical basis for the theory of "acute Q $i$ deficiency" for HAP caused by MDRO, but also enrich the theory and practice of treating infectious diseases with TCM. Additonally, it is important to form a clinical diagnosis and treatment plan or guidance for comprehensive treatment of MDRO HAP with TCM, so as to reduce the medical and financial burdens caused by infections.

\section{Trial status}

Protocol version 1, 11 February 2018.The recruitment commenced in July 2018 and aims to enroll 200 participants for the trial. It is anticipated that recruitment will end by June 2021.

\section{Supplementary information}

Supplementary information accompanies this paper at https://doi.org/10. 1186/s13063-019-3927-x

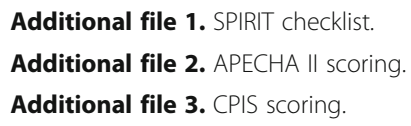

Abbreviations

CPIS: Clinical pulmonary infection score; CRF: Case report form; eCRF: Electronic case report form; EDC: Electronic data capture; HAP: Hospital acquired pneumonia; ICU: Intensive care unit; MDRO: Multi-drug resistant organisms; TCM: Traditional Chinese medicine; VAP: Ventilatilator-associated pneumonia

\section{Acknowledgements}

We would like to thank the five centers participating in this study: LongHua Hospital Shanghai University of Traditional Chinese Medicine, Shanghai TCMIntegrated Hospital, Shanghai Seventh People's Hospital, Shanghai Baoshan Integrated Chinese and Western Hospital, Shanghai Pudong New Area Gongli Hospital.

\section{Authors' contributions}

DD and ZYC contributed equally to this study. BJF conceived the study and participated in its design. DD, ZYC, LYJ, JHB, LHS, and MQY participated in the design of the intervention and the development of the recruitment strategy. LYJ, GC, YG, and WZ advised on the statistical design and sample size calculation. MQY, JHB, and LHS participated in the design of the outcome measurements and assessment of the outcomes. YG and WZ will participate in data acquisition and its interpretation. DD wrote the initial draft of the manuscript. All authors read and approved the final manuscript.

\section{Funding}

This work is supported by a grant from the Foundation of Shanghai Science and Technology Committee (18401971600). The funding body has no active 
role in the design of the study, collection, analysis, and interpretation of data, and in writing the manuscript.

\section{Availability of data and materials Not applicable.}

\section{Ethics approval and consent to participate}

This study and all relevant data have been approved by the Medical Ethics Committee of Longhua Hospital Shanghai University of Traditional Chinese Medicine (number 2019LCSY011). We ensure that this study is conducted in accordance with the principles of the Declaration of Helsinki and the principles of Good Clinical Practice. Central ethics approval has been confirmed from the Medical Ethics Committee of Longhua Hospital Shanghai University of Traditional Chinese Medicine (approval number 2019LCSY011) and we will not begin recruiting at other centers in the trial until local ethics approval has been obtained. This trial was registered at the China Clinical Trial Registry website under the identification number ChiCTR1900022429 on 11 June 2019. Signed informed consent forms will be obtained from all qualified participants before enrollment.

\section{Consent for publication}

Not applicable.

\section{Competing interests}

The authors declare that they have no competing interests.

\section{Author details}

'LongHua Hospital Shanghai University of Traditional Chinese Medicine, NO.725 Wanping South Road, Xuhui District, Shanghai 200032, China. ${ }^{2}$ The Second Clinical Medical College, Henan University of Traditional Chinese Medicine, NO.6 Dongfeng Road, Zhengzhou 450046, Henan, China. ${ }^{3}$ Shanghai Municipal Hospital of Traditional Chinese Medicine, No. 274 Zhijiang Middle Road, Jing'an District, Shanghai 200071, China. ${ }^{4}$ Department of Liver Disease, Shaanxi Provincial Hospital of Traditional Chinese Medicine, NO.4 xihuamen, Lianhu District, Xi'an 710003, Shaanxi, China. ${ }^{5}$ Shanghai Seventh People's Hospital, NO.358 Datong Road, Gaoqiao, Pudong New District, Shanghai 200137, China

\section{Received: 14 August 2019 Accepted: 22 November 2019}

Published online: 30 December 2019

\section{References}

1. Xie J, Yang Y, Huang Y, et al. The current epidemiological landscape of ventilator-associated pneumonia in the intensive care unit: A multicenter prospective observational study in China. Clin Infect Dis. 2018;67(suppl_2): S153-61. https://doi.org/10.1093/cid/ciy692.

2. Millot G, Voisin B, Loiez C, et al. The next generation of rapid point-of-care testing identification tools for ventilator-associated pneumonia. Ann Trans Med. 2017;5(22):451. https://doi.org/10.21037/atm.2017.11.05.

3. Fernández-Barat $L$, Ferrer $M$, De Rosa $F$, et al. Intensive care unitacquired pneumonia due to Pseudomonas aeruginosa with and without multidrug resistance. J Infect. 2017;74(2):142-52. https://doi.org/ 10.1016/j.jinf.2016.11.008.

4. Delle Rose D, Pezzotti P, Fortunato E, et al. Clinical predictors and microbiology of ventilator-associated pneumonia in the intensive care unit: a retrospective analysis in six Italian hospitals. Eur J Clin Microbiol Infect Dis. 2016;35(9):1531-9. https://doi.org/10.1007/s10096-016-2694-9.

5. Martischang R, Buetti N, Balmelli C, et al. Nation-wide survey of screening practices to detect carriers of multi-drug resistant organisms upon admission to Swiss healthcare institutions. Antimicrob Resist Infect Control. 2019;8(1):37. https://doi.org/10.1186/s13756-019-0479-5.

6. Gysin DV, Cookson B, Saenz H, et al. Variability in contact precautions to control the nosocomial spread of multi-drug resistant organisms in the endemic setting: a multinational cross-sectional survey. Antimicrob Resist Infect Control. 2018;7(1):81. https://doi.org/10.1186/s13756-018-0366-5.

7. Magira EE, Islam S, Niederman MS. Multi-drug resistant organism infections in a medical ICU: Association to clinical features and impact upon outcome. Med Intensiva. 2018;42(4):225-34. https://doi.org/10. 1016/j.medin.2017.07.006.

8. Laszkowska M, Terry M, Whittier S, et al. Tu1399 timing and risk factors for gastrointestinal acquisition of multi-drug resistant organisms in the intensive care unit. Gastroenterology. 2016;150(4):S894-5. https://doi.org/10. 1016/S0016-5085(16)33024-4

9. BangJiang $F$, Jianhong B. Study of traditional Chinese medicine and western medicine on septic shock. J Pract Shock. 2017;1(1):16-20,23 http://www. syxkzz.net/CN/Y2017/N1//1/16.

10. Fang BJ, Sun $\mathrm{LH}, \mathrm{Bu} J \mathrm{H}$, et al. The discussion of acute deficiency syndrome theory and its clinical application in emergency medicine (I). J Emerg Tradit Chinese Med. 2017;26(10):1724-6. https://doi.org/10.3969/j.issn.1004-745X. 2017.10.010.

11. Fang BJ, Sun $L H, B u J H$, et al. The discussion of acute deficiency syndrome theory and its clinical application in emergency medicine (II). J Emerg Tradit Chinese Med. 2017;26(11):1943-6. https://doi.org/10.3969/j.issn.1004-745X. 2017.11.017.

12. Fang BJ, Sun $L H, B u J H$, et al. The discussion of acute deficiency syndrome theory and its clinical application in emergency medicine (III). J Emerg Tradit Chinese Med. 2017;26(12):2116-7. https://doi.org/10.3969/j.issn.1004745X.2017.12.013.

13. Jiang $X$, Chen G, Huang J, et al. Modified Buzhong Yiqi decoction for myasthenia gravis: A systematic review protocol. Medicine (Baltimore). 2018 ; 97(50). https://doi.org/10.1097/MD.0000000000013677.

14. Wang DJ. Clinical application of Buzhong Yiqi decoction. J Tradit Chinese Med. 2010;51(S2):88-9. https://doi.org/10.13288/j.11-2166/r.2010.s2.183.

15. Nawen L. Examples of treatment of Buzhong Yiqi decoction. J Tradit Chinese Med. 2008;(01):93. https://doi.org/10.13288/j.11-2166/r.2008.01.046.

16. Zhang LP, Fu ZL, Zhang DB, et al. Effect of Buzhong Yiqi decoction on the body temperature of fever animals invaded by Qi deficiency. J Tradit Chinese Med. 1998;(04):236-8. 196. https://doi.org/10.13288/j.11-2166/r.1998. 04.027.

17. Chen H, Wang YH, Fang BJ, et al. Clinical study of Quyu Jiedu Yiqi decoction in the treatment of severe sepsis. Acta Universitatis Traditionis Medicalis Sinensis Pharmacologiaeque Shanghai. 2008;22(2):30-1. https:// doi.org/10.16306/j.1008-861x.2008.02.009.

18. Pan XM, Lin XF. Treatment of 31 cases of stroke complicated with hospital acquired pneumonia with Western medicine and Buzhong Yiqi decoction. J Fujian Univ Trad Chinese Med. 2013;23(5):61-2. https://doi.org/10.13261/j. cnki.jutcm.002868.

19. Zhao P, Guo DF, Lu CC, et al. Fang Bangjiang's experience in treating exogenous fever. Shanghai J Traditional Chinese Med. 2015;49(7):25-7. https://doi.org/10.16305/j.1007-1334.2015.07.008.

20. Hu B, An HM, Shen KP. Pharmacological study of Buzhong Yiqi Decoction: a review. Zhong Xi Yi Jie He Xue Bao. 2008;6(7):752-5. https://doi.org/10.3736/ jcim20080718.

21. Guo X, Mao P, Fu SG, et al. Investigation of compatibility influence on the immuno-pharmacological effects of Buzhong Yiqi decoction. Chinese J Exp Tradit Med Formulae. 1999;(01):32-5. https://doi.org/10.13422/j.cnki.syfjx. 1999.01.012.

22. Tang B, Wu MY. Advances in immunopharmacology of Buzhong Yiqi Decoction. Chinese Trad Patent Med. 1995;(01):42-3 https://kns.cnki.net/ KCMS/detail/detail.aspx?dbcode=CJFO\&dbname=CJFD9495\&filename= ZCYA501.022\&uid=WEEVRECWSIJHSIdRa1FhdkJkVG5ha1RXNERDb1 pMMW8vdVRRQkEyMy9CYz0=\$9A4hF_YAuvQ5obgVAqNKPCYCEjKensW4 IQMovwHtwkF4VYPoHbKxJw!!\&v=MTIwMjVoQVU0amg0T1g2 VHJIMDNIYk9kUkX1ZVI1ZHVGeTdnV2c9PVB5N1NiN2 EOSDgvTXJZMHFGNTRPZmdnNXo=

23. Julious SA. Sample size of 12 per group rule of thumb for a pilot study. Pharm Stat. 2005;4(4):287-91. https://doi.org/10.1002/pst.185.

24. Xu A, Zheng B, Xu YC, et al. National epidemiology of carbapenem-resistant and extensively drug-resistant Gram-negative bacteria isolated from blood samples in China in 2013. Clin Microbiol Infect. 2016;22 Suppl 1:S1-8. https://doi.org/10.1016/..cmi.2015.09.015.

25. Boyle DP, Zembower TR. Epidemiology and management of emerging drug-resistant gram-negative bacteria: extended-spectrum $\beta$-lactamases and beyond. Urol Clin North Am. 2015;42(4):493-505. https://doi.org/10.1016/j. ucl.2015.05.005.

26. Lynch BL, Schaffer K. Can guidelines for the control of multi-drug resistant gram negative organisms be put into practice? A national survey of guideline compliance and comparison of available guidelines. J Hosp Infect. 2019:1-7. https://doi.org/10.1016/j.jhin.2018.12.017.

27. Chellapandi K, Dutta TK, Sharma I, et al. Prevalence of multi drug resistant enteropathogenic and enteroinvasive Escherichia coli isolated from children with and without diarrhea in Northeast Indian 
population. Ann Clin Microbiol Antimicrob. 2017;16(1):49. https://doi.org/ 10.1186/s12941-017-0225-X.

28. Rubio-Perez I, Martin-Perez E, Domingo-García D, et al. Specific clinical profile and risk factors for mortality in general surgery patients with

infections by multi-drug-resistant Gram-negative bacteria. Surg Infect. 2017; 18(5):625-33. https://doi.org/10.1089/sur.2016.255.

\section{Publisher's Note}

Springer Nature remains neutral with regard to jurisdictional claims in published maps and institutional affiliations.

Ready to submit your research? Choose BMC and benefit from:

- fast, convenient online submission

- thorough peer review by experienced researchers in your field

- rapid publication on acceptance

- support for research data, including large and complex data types

- gold Open Access which fosters wider collaboration and increased citations

- maximum visibility for your research: over $100 \mathrm{M}$ website views per year

At $\mathrm{BMC}$, research is always in progress.

Learn more biomedcentral.com/submissions 